International Journal of Pure and Applied Mathematics

Volume 114 No. 3 2017, 445-456

ISSN: 1311-8080 (printed version); ISSN: 1314-3395 (on-line version)

url: http://www.ijpam.eu

doi: 10.12732 /ijpam.v114i3.3

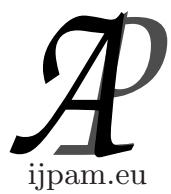

\title{
FREE CONVECTIVE AND OSCILLATORY FLOW OF A DUSTY FLUID THROUGH A POROUS MEDIUM
}

\author{
M. Vidhya ${ }^{1}$, N. Niranjana ${ }^{2}$, A. Govindarajan ${ }^{3} \S$ \\ ${ }^{1}$ Department of Mathematics, INDIA \\ Sathyabama University, Sholinganallur \\ ${ }^{2}$ Research Scholar, Sathyabama University \\ Department of Mathematics, INDIA \\ CSI Ewarts Women's Christian College \\ ${ }^{3}$ Department of Mathematics \\ SRM University, Kattankolathur, INDIA
}

\begin{abstract}
The objective of this paper is to study the effect of non-constant 2-dimensional free convective flow during the motion of a viscous incompressible dusty fluid through a highly porous medium. The porous medium is bounded by a vertical plane surface of constant temperature. The surface absorbs the dusty fluid with constant velocity and the free stream velocity of the fluid vibrates about a mean constant value. Analytical expressions for the velocity of the fluid and dust are given. The effects of Grashof number and permeability parameter upon the velocity field are also shown in a graphical representation. The velocity profiles decreases with an increase in the mass concentration of the dust particles (or) increase of frequency parameter (or) increase of permeability parameter (or) increase of Grashof number.
\end{abstract}

AMS Subject Classification: 76Txx

Key Words: dusty fluid, oscillatory flow, free convection, porous medium, relaxation time parameter, dust particles, temperature

Received: October 17, 2016

Revised: $\quad$ March 14, 2017

Published: $\quad$ May 23, 2017

$\S_{\text {Correspondence author }}$ (c) 2017 Academic Publications, Ltd. url: www.acadpubl.eu 


\section{Introduction}

The flow through a porous medium, under the influence of temperature differences, is one of the most considerable and contemporary subjects, because it finds great applications in geothermic, geo physics and technology. The study of the flow of dusty fluids is of practical importance, particularly the flow through packed beds, sedimentation, environmental pollution and centrifugal separation of particles. Considering the blood as a two-phase fluid, this study also gives some insight into the flow of blood through veins and arteries. In recent years, the requirement of modern technology has stimulated interest in fluid flow studies, which involve the interaction of several phenomena. One such study is related to the effects of free convection flow through a porous medium which plays an important role in agricultural engineering, petroleum industries and heat transfer. The convection problem in a porous medium also has important applications in geothermal reservoirs and geothermal energy extractions. Raptis et al. [1] has discussed about the influence of free convective steady flow of the viscous fluid through the porous medium, when there is constant heat flux. Acharya et al. [2] have studied the effect of porosity of the medium coupled with the variation of plate temperature with regard to space and in time. Singh and Alphonsa Mathew [3] gave theoretical analysis of the effects of permeability and the injection/suction on an oscillatory free convective flow of a viscous incompressible fluid through a highly porous medium bounded between two infinite vertical porous plates. Manglesh and Gorla [4] have analyzed the MHD free convective flow through porous medium in the presence of hall current, radiation and thermal diffusion. Kannan and Venkataraman [5] discussed about free convection in an infinite porous dusty medium induced by pulsating point heat source. Govindarajan et al. [6] analyzed the 3D couette flow of dusty fluid with transpiration cooling.Vidhya et al. [7] studied about Laminar convection through porous medium between two vertical parallel plates with heat source. Singh et al. [8] analyzed the oscillatory couette flow through porous medium in rotating system. Das et al. [9] investigated the effect of heat source on MHD free convection flow past an oscillating porous plate in the slip flow regime. Unsteady flow of a dusty fluid between two parallel plates bounded above by porous medium was discussed by Parul Saxena et al. [10]. Ramesh et al. [11] investigated the convective heat transfer in a dusty fluid over a vertical permeable surface with thermal radiation. Govindarajan et al. [12] discussed the effects of chemical reaction on unsteady MHD free convective flow in a rotating porous medium with mass transfer. Dusty fluid flows has its importance in many applications like wastewater treatment, power plant pip- 
ing, combustion and petroleum transport. The flow of fluids through porous media has become an important topic because of recovery of crude oil from pores of reservoir rocks. Several authors have examined this type of problem theoretically in various ways. Om Prakash et al. [13] analyzed about the heat transfer to MHD oscillatory dusty fluid flow in a channel filled with a porous medium. Acharya et al. [14] studied the effect of Magnetic field on the free convective and mass transfer flow through a porous medium with constant suction and constant heat flux. Raptis and Perdikis analyzed the flow through a porous medium by the presence of free convective flow [15].

The purpose of this work is to study the effects of unsteady two-dimensional free convective flow during the motion of a viscous incompressible dusty fluid through a highly porous medium. The porous medium is bounded by a vertical surface of constant temperature. This surface absorbs the dusty fluid with a constant velocity and the free stream velocity of the fluid vibrates about a mean constant value. The aim of this section is to study the effect of the dust parameters on the velocity and temperature of the dusty fluid. This paper is the extension of [1] for dusty fluids.

\section{Mathematical Analysis}

We consider the unsteady two-dimensional flow through a highly porous medium which is bounded by a vertical infinite plane surface. We assume that the dusty fluid is viscous and incompressible, the surface absorbs the fluid with a constant velocity and the velocity of the fluid far away from the surface vibrates about a mean value with direction parallel to the $x^{*}$ axis. All the fluid properties are assumed constant except that the influence of the density variation with temperature is considered only in the body force term. The $x^{*}$ axis is taken along the plane surface with direction opposite to the direction of gravity and the $y^{*}$ axis is taken to be normal to the surface.

The equations which govern the problem when the velocity and the temperature are functions of $y^{*}$ and time $t^{*}$ are:

Equation of Motion for Fluid Phase

$$
\begin{gathered}
\frac{\partial v^{*}}{\partial y^{*}}=0 \\
\frac{\partial u^{*}}{\partial t^{*}}+v^{*} \frac{\partial u^{*}}{\partial y^{*}}=-\frac{1}{\rho} \frac{\partial p^{*}}{\partial x^{*}}-g+\nu \frac{\partial^{2} u^{*}}{\partial t^{*} 2}-\frac{\nu u^{*}}{K^{*}}+\frac{K N_{0}}{\rho}\left(u_{p}^{*}-u^{*}\right)
\end{gathered}
$$




$$
\frac{\partial T^{*}}{\partial t^{*}}+v^{*} \frac{\partial T^{*}}{\partial y^{*}}=\frac{k}{\rho c_{p}} \frac{\partial^{2} T^{*}}{\partial y^{2 *}}+\frac{N o m C_{s}}{\rho c_{p} \tau_{p}}\left(T_{p}^{*}-T^{*}\right)
$$

Equation of Motion for Particle Phase

$$
\begin{gathered}
\frac{\partial u_{p}^{*}}{\partial y^{*}}=0 \\
\frac{\partial u_{p}^{*}}{\partial t^{*}}+v_{p}^{*} \frac{\partial u_{p}^{*}}{\partial y^{*}}=\frac{K}{m}\left(u^{*}-u_{p}^{*}\right) \\
\frac{\partial T_{p}^{*}}{\partial t^{*}}+v_{p}^{*} \frac{\partial T_{p}^{*}}{\partial y^{*}}=-\frac{\left(T_{p}^{*}-T^{*}\right)}{\tau_{p}}
\end{gathered}
$$

\section{Boundary Conditions}

$$
\begin{aligned}
& y^{*}=0: u^{*}=0, u_{p}^{*}=0, v^{*}=-v_{0}=\text { constant, } v_{p}^{*}=v_{0}, T_{p}^{*}=T_{w}^{*}, T^{*}=T_{w}^{*} \\
& y^{*} \rightarrow \infty^{0} u^{*}=U\left(1+\epsilon e^{i \omega^{*} t^{*}}\right), T^{*} \rightarrow T \infty^{*}, \\
& u p^{*}=U^{*} \rightarrow U\left(1+\epsilon e^{i \omega^{*} t^{*}}\right), T_{p}^{*} \rightarrow T_{\infty}^{*}
\end{aligned}
$$

equation (2) for free stream, is reduced to $\rho \frac{\partial u^{*}}{\partial t^{*}}=-\frac{\partial p}{\partial x^{*}}-\rho_{\infty} g-\frac{u}{K^{*}} U^{*}$

On eliminating $\frac{\partial p}{\partial x^{*}}$ between (2) and (8) and using the constitutive equation $\left(\rho_{\infty}-\rho\right)=\beta \rho\left(T^{*}-T \infty^{*}\right)$ we have

$$
\begin{aligned}
& \left(\frac{\partial u^{*}}{\partial t^{*}}-v_{0} \frac{\partial u^{*}}{\partial y^{*}}\right) \\
& \quad=\frac{\partial U^{*}}{\partial t^{*}}+g \beta\left(T^{*}-T \infty^{*}\right)+\nu \frac{\partial^{2} u^{*}}{\partial y^{* 2}}+\frac{\nu}{K^{*}}\left(U^{*}-u^{*}\right)+\frac{K N 0}{\rho}\left(u_{p}^{*}-u^{*}\right)
\end{aligned}
$$

we introduce the non dimensional variables

$$
\begin{aligned}
& u=\frac{u^{*}}{U}, u_{p}=\frac{u_{p}^{*}}{U}, t=\frac{t^{*} v_{0}^{2}}{\nu}, y=\frac{y^{*} v_{0}}{\nu}, \\
& U_{0}=\frac{U^{*}}{U}, \omega=\frac{v \omega^{*}}{v_{0}^{2}}, T=\frac{T^{*}-T \infty^{*}}{T w^{*}-T \infty^{*}}, T_{p}=\frac{T p^{*}-T \infty^{*}}{T w^{*}-T \infty^{*}} \\
& \operatorname{Pr}=\frac{\rho \nu c_{p}}{k}, G r=\frac{v g \beta\left(T w^{*}-T \infty^{*}\right)}{V v_{0}^{2}}, k_{0}=\frac{v_{0}^{2}}{\nu^{2}} K^{*}, f=\frac{N_{0} m}{\rho} \\
& \Lambda=\frac{\tau_{p} V_{0}^{2}}{\nu}, \gamma=\frac{c_{s}}{c_{p}}
\end{aligned}
$$


The above equations are reduced to the following non-dimensional form.

$$
\begin{gathered}
\frac{\partial u}{\partial t}-\frac{\partial u}{\partial y}=\frac{\partial u_{0}}{\partial t}+\frac{\partial^{2} u}{\partial y^{2}}+G r T+\frac{\left.\partial U_{0}-u\right)}{k_{0}}+\frac{f}{\Lambda}\left(u_{p}-u\right) \\
\operatorname{Pr}\left(\frac{\partial T}{\partial t}-\frac{\partial T}{\partial y}\right)=\frac{\partial^{2} T}{\partial y^{2}}+\frac{2 f}{3 \Lambda}\left(T_{p}-T\right) \\
\frac{\partial u_{p}}{\partial t}+\frac{\partial u_{p}}{\partial y}=\frac{1}{\Lambda}\left(u-u_{p}\right) \\
\frac{\partial T_{p}}{\partial t}+\frac{\partial T_{p}}{\partial y}=\frac{1}{\Lambda}\left(T-T_{p}\right)
\end{gathered}
$$

The boundary conditions reduce to

$$
\begin{aligned}
& y=0: u=0, u_{p}=0, T=1, T_{p}=1 \\
& y \rightarrow \infty: u \infty\left(1+\epsilon e^{i \omega t}\right), u_{p} \rightarrow\left(1+\epsilon e^{i \omega t}\right), T \rightarrow 0, T_{p} \rightarrow 0
\end{aligned}
$$

In order to solve the differential equations (10) to (13) we assure that, $f(y, t)=f_{0}(y)+\epsilon e^{i \omega t} f_{1}(y)+\ldots$

$$
\text { where } f \text { is replaced by } u, u_{p}, T, T_{p} \text {. }
$$

substituting (15) in (10) to (13) we get the following system of differential equations

$$
\begin{gathered}
u_{0}^{11}+u_{0}^{1}-\frac{u_{0}}{k_{0}}+\frac{f}{\Lambda}\left(u_{p_{0}}-u_{0}\right)=-\frac{1}{k_{0}}-G r T_{0} \\
(\Lambda D+1) u_{p 0}=u_{0} \\
T_{0}^{11}+P_{r} T_{0}^{1}+\frac{2 f}{3 \Lambda}\left(T_{p 0}-T_{0}\right)=0 \\
(\Lambda D+1) T_{p 0}=T_{0} \\
u_{1}^{11}+u_{1}^{1}-u_{1}\left(i \omega+\frac{1}{k_{0}}\right)+\frac{f}{\Lambda}\left(u_{p 1}-u_{1}\right)=-G r T_{1}-\left(\frac{1}{k_{0}}+i \omega\right)
\end{gathered}
$$




$$
\begin{gathered}
i \omega u_{p 1}+\frac{\partial u p_{1}}{\partial y}=\frac{\left(u_{1}-u_{p 1}\right)}{\Lambda} \\
T_{1}^{11}+\operatorname{Pr} T_{1}^{1}-i \omega \operatorname{Pr} T_{1}+\frac{2 f}{3 \Lambda}\left(T_{p 1}-T_{1}\right)=0 \\
i \omega T_{p 1}+T p_{1}^{2}=\frac{\left(T_{1}-T_{p 1}\right)}{\Lambda}
\end{gathered}
$$

with corresponding boundary conditions

$$
\begin{array}{r}
y=0: u_{0}=0, u_{1}=0, u_{p 0}=0, u_{p 1}=0, T_{0}=1, T_{1}=0, T_{p 0}=1, T_{p 1}=0 \\
y \rightarrow \infty: u_{0} \rightarrow 1, u_{1} \rightarrow 1, u_{p 0} \rightarrow 0, u_{p 1} \rightarrow 1, T_{0} \rightarrow 0, T_{1} \rightarrow 0, T_{p 0} \rightarrow 0, T_{p 1} \rightarrow 0
\end{array}
$$

solving the differential equations (16) to (23) using the boundary condition (24) we get

$$
\begin{gathered}
T_{0}=e^{-m y}, T_{p 0}=\left(\frac{e^{-m y}-m \Lambda e^{-\frac{y}{\Lambda}}}{1-m \Lambda}\right), \\
u_{0}=1-(G r P I+1) e^{R_{1} Y}+e^{-m y} G r P I, \\
u_{p 0}=\frac{(G r P I+1)}{\left(1+R_{1} \Lambda\right)}\left(e^{-\frac{y}{\Lambda}}-e^{R_{1} y}\right)+\frac{G r P I}{1-\Lambda m}\left(e^{-m y}-e^{-\frac{y}{\Lambda}}\right)+\left(1-e^{-\frac{y}{\Lambda}}\right), \\
T_{1}=0, T_{p 1}=0, \\
u_{1}=\left(1-e^{R} 3^{y}\right), \\
u_{p 1}=\frac{1}{1+i \Lambda \omega+R_{3} \Lambda}\left[e^{-\frac{(1+i \Lambda \omega) y}{\Lambda}}-e^{R_{3} y}\right]+1-e^{-\left(\frac{(1+i \Lambda \omega)}{\Lambda}\right)^{y}},
\end{gathered}
$$

where $m, R_{1}, R_{3}, P I$ are given by

$$
\frac{m=(\operatorname{Pr} \Lambda+1)+\sqrt{(\operatorname{Pr} \Lambda+1)^{2}-4 \Lambda\left(\operatorname{Pr}-\frac{2 f}{3}\right)}}{2 \Lambda}
$$




$$
\begin{gathered}
R_{1}=-\frac{-\left(1-f-\frac{\Lambda}{k_{0}}\right)-\sqrt{\left(1-f-\frac{\Lambda}{k_{0}}\right)^{2}+\frac{4(\Lambda+1)}{k_{0}}}}{2(\Lambda+1)} \\
P I=\frac{-(1-\Lambda m)}{-\Lambda m^{3}+(\Lambda+1) m^{2}-m\left(1-f-\frac{\Lambda}{k_{0}}\right)-\frac{1}{k_{0}}} \\
R_{3}=\frac{-\left(1-f-\frac{\Lambda}{\mathrm{k}_{0}}\right)-\sqrt{\left(1-f-\frac{\Lambda}{\mathrm{k}_{0}}\right)^{2}+4[1+\Lambda(1+i \omega)]\left[(1+i \Lambda \omega)\left(\frac{1}{\mathrm{k}_{0}}+i \omega\right)+i \omega f\right]}}{2[1+\Lambda(1+i \omega)]}
\end{gathered}
$$

\section{Result and Discussion}

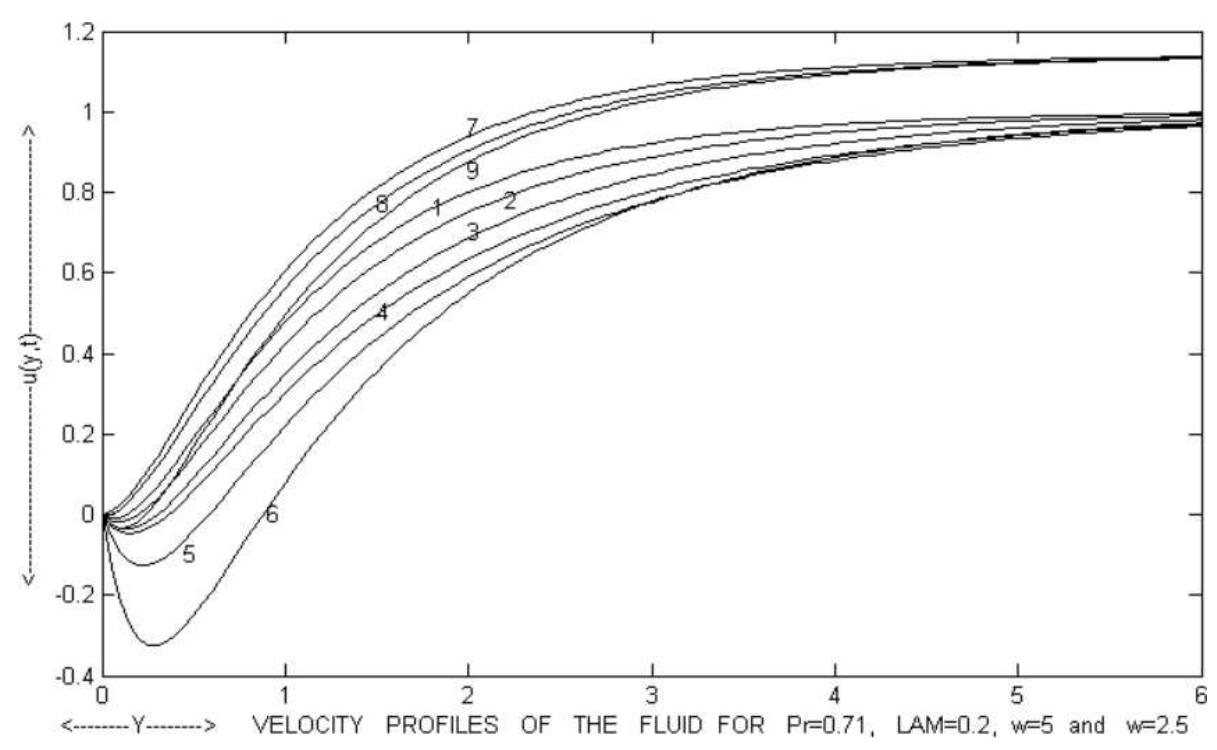

Figure 1: Velocity Profiles of the fluid for $\operatorname{Pr}=0.71 \Lambda=0.2 \omega=5$

The velocity profiles of the fluid and that of the dust are plotted in fig-1 \& fig-2 respectively. From fig-1 \& fig- 2 it is clear that both the fluid and the dust behave in the same manner. Both the profiles decrease with an increase in the mass concentration of the dust particles (or) increase of the frequency parameter (or) increase of the permeability parameter (or) increase of Grashof number. All the profiles of both the fluid and dust show a slow decrease (retardation) 
near the lower plate and increase steadily thereafter and reach the value 1 at the other plate. For large values of the Grashof number and permeability parameter the retardation (slow decrease) is more near the lower plate in the case of the fluid profiles than in the case of the dust profiles.

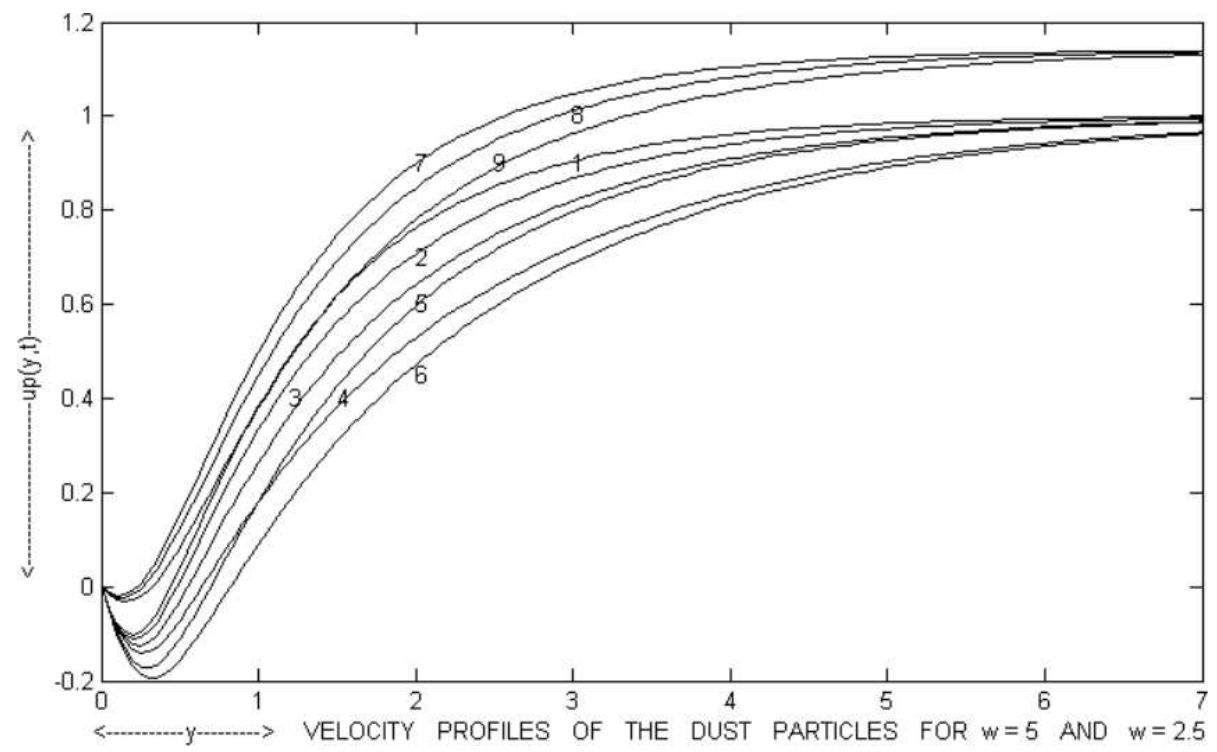

Figure 2: Velocity Profiles of the dust particles for $\operatorname{Pr}=0.71 \Lambda=0.2$ $\omega=5$

Table 1: Table for fig-01 \& fig-02

\begin{tabular}{|c|c|c|c|c|}
\hline Curve & $G r$ & $K_{0}$ & $\omega$ & $f$ \\
\hline 1 & 3 & 3 & 5 & 0.2 \\
\hline 2 & 3 & 3 & 5 & 0.4 \\
\hline 3 & 3 & 5 & 5 & 0.2 \\
\hline 4 & 3 & 5 & 5 & 0.4 \\
\hline 5 & 5 & 5 & 5 & 0.2 \\
\hline 6 & 5 & 5 & 5 & 0.4 \\
\hline 7 & 3 & 3 & 2.5 & 0.2 \\
\hline 8 & 3 & 3 & 2.5 & 0.4 \\
\hline 9 & 3 & 5 & 2.5 & 0.2 \\
\hline
\end{tabular}

The decrease is more in the case of the fluid profiles when compared with 
that of the dust profiles. Differences of the velocity are greater in the case of the dust particles than the dusty fluid when there is an increase in the mass concentration of the dust particles.

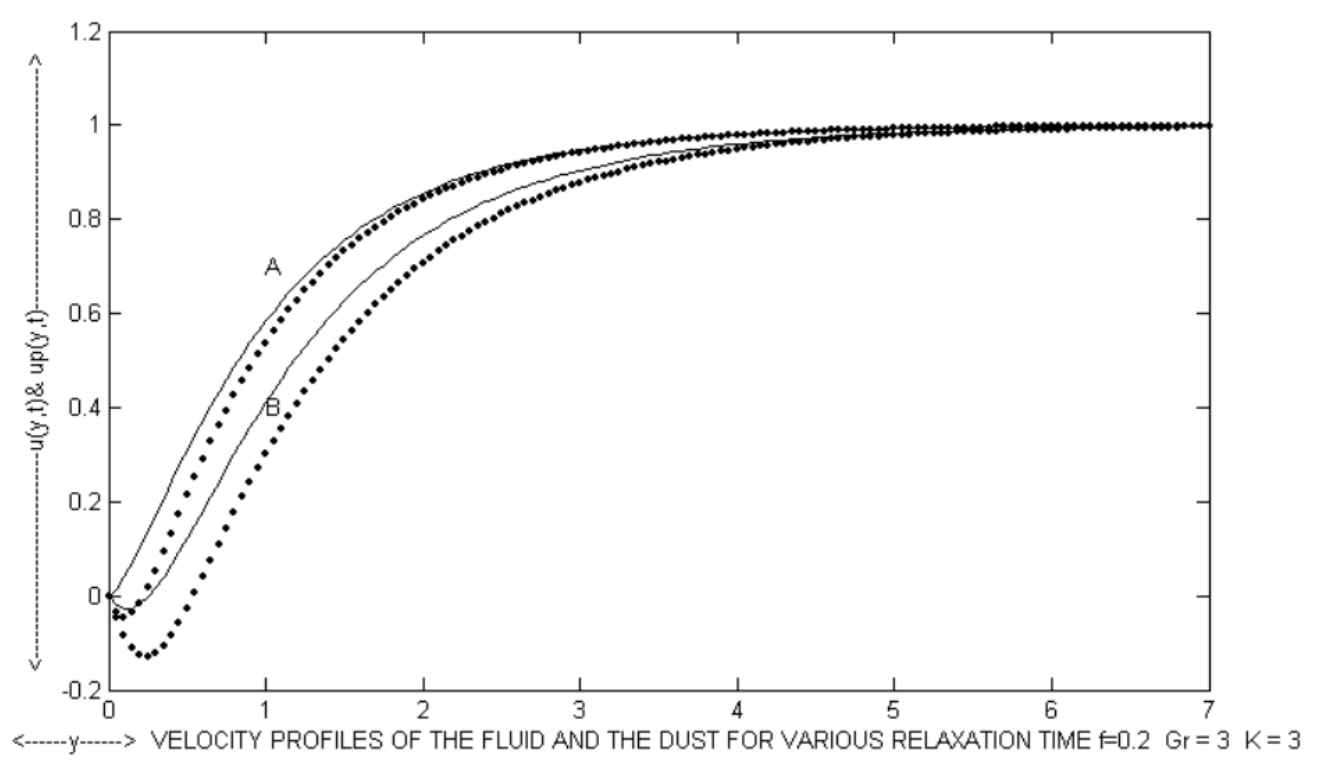

Figure 3: Velocity Profiles of the fluid \& dust particles for $\operatorname{Pr}=0.71$, $f=0.2, \omega=0.5, k_{0}=3 \&$ various values of relaxation time, $A \rightarrow \Lambda=$ $0.10, B \rightarrow \Lambda=0.11$, curve represents fluid, dotted curve represents dust.

Fig-3 shows the velocity profiles of the fluid and dust for various relaxation time of the dust particles. The velocity of the fluid is represented by curve and velocity of the dust by dotted curve. For $G r=3, k_{0}=3, \operatorname{Pr}=0.71$, respectively, ' $A$ ' represents the profiles for relaxation time $0.1 \&$ ' $B$ ' represents the profiles for relaxation time 0.11 .

It is clear from fig-3 that the velocity profiles of both fluid \& dust decrease when there is an increase in the relaxation time of the dust particles. For small values of the relaxation time the difference in velocity between the fluid and dust is very small.

As relaxation time increases, the difference in velocity between the fluid and dust also increase. Both the profiles show slight decrease near the lower plate and there after increase steadily \& becomes ' 1 ' at the other plate. For both the fluid \& dust the amount of decrease is more when the relaxation time of the dust particles increases. 


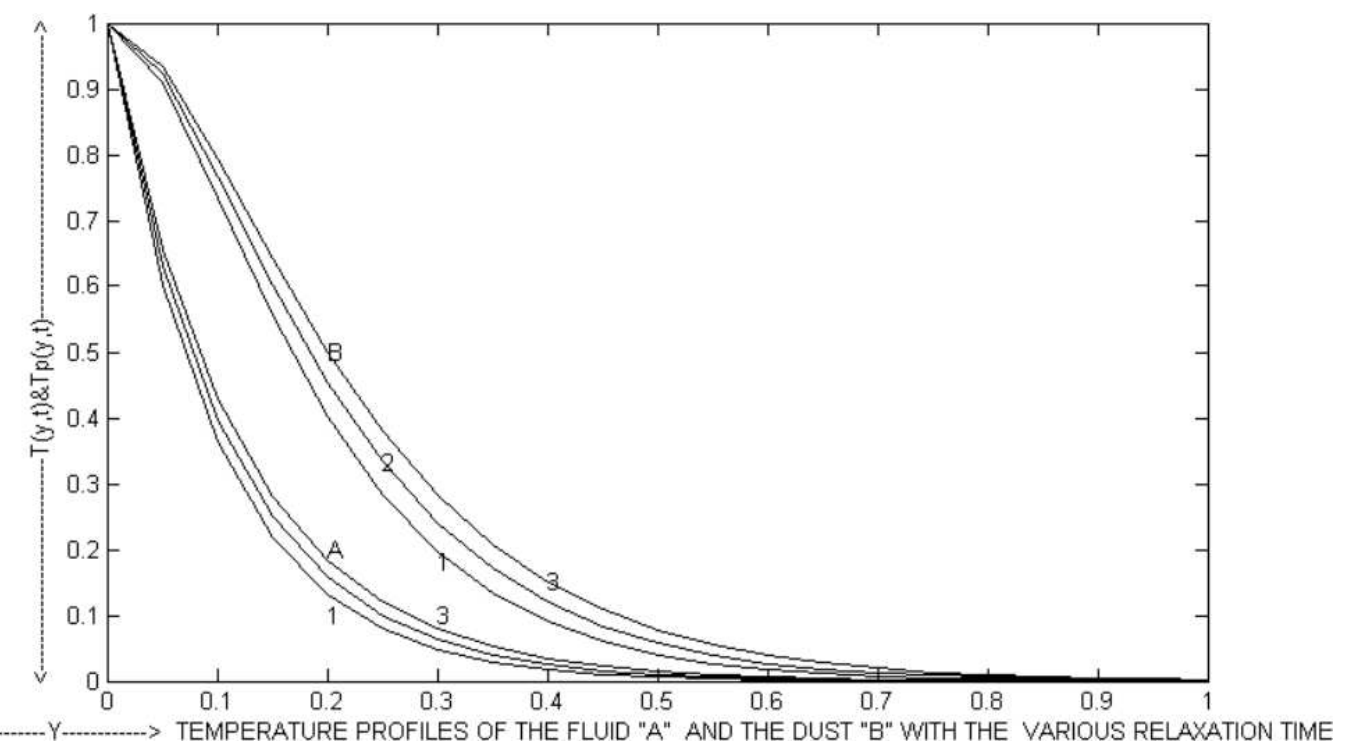

Figure 4: Temperature profiles of the fluid ' $A$ ' \& ' $B$ ' that of dust, for $f=0.2, G r=3, k_{0}=3, \operatorname{Pr}=0.71$

\begin{tabular}{|c|c|}
\hline Curve & $\Lambda$ \\
\hline 1 & 0.10 \\
\hline 2 & 0.11 \\
\hline 3 & 0.12 \\
\hline
\end{tabular}

The temperature profiles are plotted for various values of the relaxation time and shown in fig-4. " $A$ " - represents the temperature profiles of the fluid and ' $B$ ' - represents the temperature profiles of the dust. The temperature profiles of the fluid and dust are drawn for $f=0.2, G r=3, k_{0}=3, p r=0.71$, and for various values of relaxation time of the dust particles. From fig- 4 it is clear that both the temperature profiles of the fluid and dust decrease steadily from 1 in the lower plate to zero at the other plate. Both the profiles increase with an increase in the relaxation time of the dust particles. The difference in temperature is more in the case of the dust than in the case of the dusty fluid when the relaxation time of the dust particles increases. Temperature profiles of the dust are at greater heights when compared with temperature profiles of the fluid. The temperature profiles of both the fluid and dust show a negligible change in their profiles when the mass concentration of the dust particle changes. That is the reason why the temperature profiles of both fluid and dust with various values of mass concentration of the dust particles is not 
drawn. When the dust parameters are neglected we see that our results coincide with that of $[1]$.

\section{Conclusion}

For clean fluid $(f=0)$. The velocity increases when the Grashof number and the permeability parameter increase and the difference of the velocity are greater as the Grashof number increases. But in the case of the dusty fluid there is a decreasing trend and the differences of the velocity are greater in the case of the dust particles than dusty fluid when the mass concentration of the dust particles changes.

This is due to the presence of dust particles. It is clear that the dust particle has an influence in changing the velocity of the fluids when compared with clean fluid profiles.

\section{References}

[1] A.A. Raptis, C.P. Perdikis, Oscillatory flow through a porous medium by the presence of free convective flow, International Journal of Engineering Science, 23(1) (1985), 51-55.

[2] A.K. Acharya, G.C. Dash, S.R. Mishra, Free Convective Fluctuating MHD Flow through Porous Media Past a Vertical Porous Plate with Variable Temperature and Heat Source, Hindawi Publishing Corporation Physics Research International, 2014, Article ID 587367, 8 pages.

[3] K.D. Singh, Alphonsa Mathew, An Oscillatory Free Convective Flow Through Porous Medium in a Rotating Vertical Porous Channel, Global Journal of Science Frontier Research Mathematics and Decision Sciences, 12(3) (2012).

[4] A. Manglesh, M.G. Gorla, MHD free convective flow through porous medium in the presence of hall current, radiation and thermal diffusion, Indian Journal of Pure and Applied Mathematics, 44(6) (2013), 743-756.

[5] V. Venkataraman, K. Kannan, R. Dharmarajan, A Survey of Mathematical Approaches to Two Phase Fluid Flow Techniques, Global Journal of Science Frontier Research Mathematics and Decision Sciences, 12(9) (2012).

[6] A. Govindarajan, V. Ramamurthy, K. Sundarammal, 3D couette flow of dusty fluid with transpiration cooling, Journal of Zhejiang University Science-A, 8(2), 313-322.

[7] M. Vidhya, K. Sundarammal, Laminar convection through porous medium between two vertical parallel plates with heat source, Frontiers in Automobile and Mechanical Engineering, IEEE Conference (2010), 197-200.

[8] K.D. Singh, M.G. Gorla, Hans raj, A periodic solution of oscillatory couette flow through porous medium in rotating system, Indian Journal of Pure and Applied Mathematics, 36(3) (2005), 151-159. 
[9] S.S. Das, L.K. Mishra, P.K. Mishra, Effect of heat source on MHD free convection flow past an oscillating porous plate in the slip flow regime, International Journal of Energy and Environment, 2(5) (2011), 945-951.

[10] Parul Saxena, Manju Agarwal, Unsteady flow of a dusty fluid between two parallel plates bounded above by porous medium, International Journal of Engineering, Science and Technology, 6(1) (2014), 27-33.

[11] G.K. Ramesh, B.J. Gireesha, C.S. Bagewadi, Convective Heat Transfer in a Dusty Fluid over a Vertical Permeable Surface with Thermal Radiation, International Journal of Nonlinear Science, 14(2) (2012), 243-250.

[12] A. Govindarajan, Ali. J. Chamkha, Sundarammal kesavan, M. Vidhya, Chemical reaction effects on unsteady magnetohydrodynamic free convective flow in a rotating porous medium with mass transfer, Thermal science, 18(2) (2014), s515-s526.

[13] OM Prakash, O.D. Makinde, Devendrakumar, Y.K Dwivedi, Heat transfer to MHD oscillatory dusty fluid flow in a channel filled with a porous medium, Indian Academy of Sciences, 40(4) (2015), 1273-1282.

[14] M. Acharya, G.C. Dash, L.P. Singh, Magnetic field effects on the free convection and mass transfer flow through porous medium with constant suction and constant heat flux, Indian Journal of Pure and Applied Mathematics, 31(1) (2000), 1-18.

[15] A.A. Raptis, Flow through a porous medium in the presence of a magnetic field, International Journal of Energy Research, 10(1) (1986), 97-100. 